\title{
Compte rendu de Mythes et usages des mythes de
} Bruno Saura

\section{Sophie Chave-Dartoen}

\section{OpenEdition}

1 Journals

Édition électronique

URL : http://journals.openedition.org/jso/7862

DOI : $10.4000 /$ jso.7862

ISSN : $1760-7256$

Éditeur

Société des océanistes

\section{Édition imprimée}

Date de publication : 15 décembre 2017

Pagination : 358-363

ISSN : 0300-953x

\section{Référence électronique}

Sophie Chave-Dartoen, "Compte rendu de Mythes et usages des mythes de Bruno Saura », Journal de la Société des Océanistes [En ligne], 144-145 | 2017, mis en ligne le 15 décembre 2017, consulté le 15 mars 2021. URL : http://journals.openedition.org/jso/7862 ; DOI : https://doi.org/10.4000/jso.7862

Ce document a été généré automatiquement le 15 mars 2021.

\section{(†)

Journal de la société des océanistes est mis à disposition selon les termes de la Licence Creative Commons Attribution - Pas d'Utilisation Commerciale - Pas de Modification 4.0 International. 


\title{
Compte rendu de Mythes et usages des mythes de Bruno Saura
}

\author{
Sophie Chave-Dartoen
}

\section{RÉFÉRENCE}

Saura Bruno, 2013. Mythes et usages des mythes. Autochtonie et idéologie de la Terre Mère en Polynésie, Paris-Louvain-Walpole, MA, Peeters, Selaf 444, Langues et cultures du Pacifique 18, XVIII-293 p., bibliogr., 2 cartes.

1 Le dernier livre de Bruno Saura est ambitieux à plusieurs titres. Proposant une analyse transversale des anciens mythes polynésiens de création, il offre une synthèse sur la question de l'autochtonie en Polynésie orientale et sur les mythes qui peuvent la fonder, questionnant leur validité et la validité des revendications politiques qui s'y réfèrent en contexte tahitien. Cette tentative érudite et stimulante a le mérite de formuler des interprétations très fortes qui complètent les autres publications de l'auteur sur les relations entre identité et religion d'une part (Saura, 2013, 2014a), terre et identité d'autre part (Saura, 2000, 2004, 2014b). B. Saura ouvre ainsi de nouvelles pistes de discussion et de recherche pour les spécialistes des sciences sociales comme pour les acteurs locaux auxquels il se réfère. J'en suggérerai quelques-unes ici, espérant montrer tout l'intérêt d'un ouvrage passionnant et accessible à un vaste public.

2 La question fondamentale de l'ouvrage est la suivante : comment peut-on revendiquer un lien privilégié à une terre, à un sol, dans une région où les mythes et les représentations relatifs au cosmos et au monde social ne permettent pas de le fonder en principe ? L'enjeu est ici de taille en ce qu'il a une portée politique importante. Les Polynésiens - notamment ceux de Polynésie française - peuvent-ils faire valoir des droits exclusifs sur des territoires dont ils ont été dépossédés par la colonisation? À quel point leur souveraineté peut-elle s'étendre alors que leur arrivée est historiquement récente et que des mythes documentent les migrations dont ils sont issus? Le recours à l'idée de la «terre-mère » (p. 20) et à l'histoire ancienne - en fait, à 
sa reconstruction ${ }^{1}-$, afin d'impulser un renouveau identitaire et d'en faire un levier politique pour accéder à des formes de visibilité et d'autonomie, est-il légitime dans leur cas? L'auteur construit et argumente sa réponse au fil du volume: les revendications des Polynésiens sont légitimes et recevables dans le cadre du droit international qui confère reconnaissance et droits aux peuples autochtones - parmi lesquels ils peuvent être classés du fait de leur spécificité historique et culturelle - au sein des dispositifs qu'ont mis en place les puissances coloniales, qu'il s'agisse de la République française, du Chili, de la Nouvelle-Zélande ou des États-Unis par exemple. En revanche, les revendications autonomistes radicales ne peuvent être fondées sur l'idée d'une relation consubstantielle, donc exclusive, avec le sol où ces sociétés se sont développées avant l'arrivée des Occidentaux. Si l'«autochtonie est relative dans le temps et dans l'espace» (p. 11), si «les identités autochtones ne sont pas de simples héritages mais bien des produits de l'histoire ", celles des Polynésiens et, notamment, les récits mythiques d'autochtonie des insulaires de Polynésie française sont particulièrement scrutés ici afin de "les réévaluer pour prendre en compte les conditions historiques de leur élaboration» (p. 21). L'enquête déroulée au fil des cinq chapitres ${ }^{2}$ dégage ce que peut signifier «autochtonie» en Polynésie, plus particulièrement en Polynésie française. Mythes, histoire et pratiques anciennes sont ainsi considérés afin de dénoncer les formes actuelles d'instrumentalisation politique et de plaider pour une reconnaissance mutuelle. Ce faisant, l'ouvrage offre des clés de compréhension et ouvre de nouvelles pistes de réflexion pour une reformulation des discours identitaires et politiques des peuples autochtones de la région. Il pose aussi d'intéressants problèmes de méthode et d'interprétation.

3 L'introduction rappelle utilement les aspects majeurs des problématiques liées à l'autochtonie. Bruno Saura précise les deux dimensions - étymologiquement fondées du terme "autochtone »: la première pose "la consubstantialité de la terre et de l'homme » qui y vit (p. 1) ; la seconde pose une antériorité, "souvent non datée », dans l'occupation du sol (p.2). Si, dans tous les cas, rappelle l'auteur, l'autochtonie est extrêmement difficile à définir :

«nous considérerons que les peuples autochtones existent, jusqu'à preuve du contraire, et qu'ils sont potentiellement en attente d'une définition. » (p. 12)

Si la définition de l'autochtonie pose problème, c'est que le concept offre un important levier critique, éthique et politique face à la colonisation et aux impérialismes ${ }^{3}$, mais peut avoir une toute autre portée (notamment à travers des formes de nationalisme exacerbées) dans le cadre des tensions et des conflits intercommunautaires. Se fondant sur les travaux de J.-P. Vernant, M. Détienne et N. Loreaux relatifs aux mythes grecs, l'auteur part donc en quête des mythes d'«autochtonie littérale" polynésiens qui énoncent :

« [le] rôle primordial de la terre dans la naissance de l'humanité » (p. 14),

motif quasi universel des mythes de création. Concernant la Polynésie, il décèle donc, par « décryptage ${ }^{4}$ » (p. 81), l'autochtonie littérale pour en relativiser la portée et déconstruire le discours des insulaires revendiquant l'autonomie politique. Il s'agit :

« de savoir si oui ou non, et de quelle manière, l'homme y naissait de la terre

[comme les] nationalistes et les mouvements identitaires défensifs » (p. 20)

le prétendent, mais aussi de :

« réévaluer [ces mythes dans leur usage moderne] pour prendre en compte les

dimensions historiques de leur élaboration. » (p. 21) 
$7 \quad$ Le propos est de (re-)considérer la portée politique de ces mythes en comprenant ce qui fonde leur usage et les conditions de cet usage. L'analyse du présent repose donc sur l'analyse d'un corpus ancien reconstitué à propos. Elle ne s'attache donc pas à l'étude des anciennes représentations du cosmos et de la place - du statut - qu'y trouvaient les humains dans le cadre de sociétés locales spécifiques, de leurs systèmes de représentation particuliers et de leurs évolutions, mais procède selon les méthodes de la mythologie comparée et, ponctuellement, de l'analyse structurale (S. Dunis et donc C. Lévi-Strauss sont des références importantes). Je reviendrai sur la méthode retenue par l'auteur, mais il aurait été utile qu'elle soit bien spécifiée en introduction et/ou que les niveaux de l'analyse et de la comparaison soient systématiquement identifiés (universaux, généralité et extension des motifs, coexistences) afin d'éviter d'apparents paradoxes tels "l'univers des profondeurs, à la fois solide et liquide ${ }^{5}$ " (p.121) par exemple.

8 Partant donc de la généralité de l'idée de consubstantialité entre la terre et les hommes (sens premier d'« autochtonie ») dans les mythes connus par ailleurs, l'auteur montre qu'en Polynésie la terre-matrice est, en quelque sorte, réduite à :

« [une] entité primordiale, une matière, origine de la matière » (62),

9 essentiellement passive et polymorphe, dont sont parfois arrachés ou modelés déités et êtres humains. La dimension matérielle de cette entité est opposée au principe masculin, divin et immatériel (chap.1). Il est à noter que ces mythes, très variés, souvent hétérogènes et parfois contradictoires, instaurent des chronologies et des hiérarchies parmi les entités du cosmos, notamment entre hommes et femmes. Si l'aspect matriciel de la terre, le caractère chtonien des figures féminines primordiales sont dégagés, leur sens et leur valeur sont pondérés: si les sociétés polynésiennes anciennes pensaient l'autochtonie sous une forme "littérale", c'est de façon peu cohérente, non élaborée et peu saillante (chap.1) au regard d'apports extérieurs valorisés, qu'il s'agisse du principe d'altérité sexuée (ici masculine) nécessaire dans une génération humaine socialement aboutie (chap. 2), de l'origine exogène des sociétés polynésiennes (chap.3) et de leurs aristocrates conçus comme extérieurs du fait de leurs ancêtres divins (chap. 4).

10 En effet, le caractère « inabouti » des êtres consubstantiellement attachés à la terremère montre que la vie et la fertilité proviennent de ce principe féminin, mais que ces êtres ne peuvent se développer pleinement sans l'intervention d'entités ou d'un principe masculins conçus comme supérieurs ${ }^{6}$. Ainsi Saura conclut-il le deuxième chapitre en citant Lévi-Strauss (1968) :

"L'incapacité des femmes chtoniennes polynésiennes à enfanter le multiple nous enseigne que l'autochtonie est fondamentalement négation de la capacité à la production de l'Autre, au profit d'une reproduction du Soi, du Même, qui débouche inexorablement sur la mort. L'autochtonie littérale n'est donc pas une valeur en elle-même; simplement une étape sur un chemin qui doit conduire à son dépassement, à la naissance d'une humanité parfaitement achevée. Il y a là une belle leçon à méditer pour les penseurs contemporains, polynésiens ou autres, parfois animés par le fantasme politique de l'autochtonie. » (p. 105)

11 Le propos du livre prend un tournant au troisième chapitre dont l'incipit (p. 107) est une citation de Ph. Bachimon :

«Les Māohi [Polynésiens] ne se conçoivent pas comme autochtones de leur île. » $(1995: 234)$ 

mythes. Évaluant

«le poids d'une longue culture de l'errance et de la navigation dans l'organisation sociale, politique et religieuse des sociétés [...]»(p. 23),

l'auteur entame une "archéologie des mythes »(p.112), recherchant, après Serge Dunis :

«les fondements austronésiens du patrimoine cosmologique et légendaire des Polynésiens » (p. 113),

notamment les mythes d'origine démontrant leur caractère exogène. Sont également abordées ici :

« des scènes de pêche et de conquête des îles, qui relativisent [...] considérablement

l'affirmation de l'autochtonie polynésienne. » (125)

$\mathrm{Au}$ quatrième chapitre, la perspective historique se détourne des questions de migration pour s'attacher à savoir si «les Polynésiens ont cru à leurs mythes». L'ethnologie est cette fois convoquée pour montrer que, d'une part, l'organisation sociale et le mode de vie insulaire contribuaient au «sentiment pour ceux qui y résident, d'une intimité avec la terre » (p. 160), tandis que, d'autre part, l'idéologie fondant les inégalités sociales affirmait, face à "l'antériorité, l'intériorité, l'infériorité " (184) de la population locale, «l'étrangeté, la "nature" différente des chefs polynésiens, venus d'un ailleurs supérieur» (181) car divin. Le dernier développement, enfin (chap. 5), donne une lecture politiste de l'histoire récente de ces îles, des formes de colonisation, de dépossessions, parfois même d'anéantissement imposées aux populations insulaires, passant par la médiation des femmes et la dévirilisation des hommes. Il revient aussi sur les enjeux que constituent la terre, la souveraineté politique et la défense de l'environnement pour les indépendantistes et les adeptes du renouveau culturel. À travers les métaphores de l'île maternelle et de l'enracinement ainsi que la réinterprétation des mythes anciens, ils défendent leur « autochtonie » (et l'usage récent du terme mā'ohi pour la désigner) ainsi que :

« les liens unissant l'homme Polynésien [sic] à sa "terre-mère", opposée à la "mèrepatrie" d'emprunt qu'est la puissance coloniale française. » (p. 203)

Le livre finit donc sur les représentations contemporaines relatives aux mythes anciens, à la terre, à la femme, et aux usages qui en sont faits. En résumé (pages 252-254 de la conclusion), la terre, principe d'où émerge l'humanité, n'a jamais fait l'objet d'un culte spécifique en Polynésie. Les mythes polynésiens ne correspondent aussi que partiellement au schéma classique de l'autochtonie littérale, et

« la naissance des humains à partir de la terre n'a pas de conséquence nécessaire en matière d'appropriation d'un territoire particulier.» (p. 254)

Il faut enfin considérer les «idéologies religieuses et conquérantes " qui valorisaient autrefois une filiation lointaine et divine pour les hommes de haut statut qui entretenaient :

«une distance physique et symbolique avec toutes les entités intérieures et inférieures, desquelles participait la terre.» (p. 254)

Ces faits historiquement attestés invalident, selon l'auteur, toute revendication à des formes anciennes de consubstantialité avec la terre pour obtenir la reconnaissance de droits politiques. Le livre propose, in fine, une position éthique en écho aux préoccupations contemporaines sur l'avenir de la planète et soutient les revendications juridiques et culturelles des autochtones : 
"le statut de ces peuples [autochtones] minoritaires, dont les descendants défendent bravement l'héritage de leur patrimoine et de leur matrimoine, passe aujourd'hui par un salut plus global de la planète, dont l'ensemble des habitants auraient sans doute intérêt à cultiver des liens charnels, des liens ombilicaux avec la terre. » (p. 262)

19 La méthode choisie et les conclusions de cet ouvrage passionnant mériteraient une discussion approfondie. Je m'en tiendrai à un regret, à quelques remarques et à des propositions. Tout d'abord, du point de vue de l'ethnologue, l'interprétation des mythes, sans considération particulière pour le corpus dont ils font partie, ni pour la signification de leurs éléments dans la société d'origine, ni encore, pour l'organisation interne de ces éléments, est déroutante, de même que l'effacement des contextes sociaux et rituels qui leur étaient associés. A minima, une brève clarification introductive sur les sources utilisées, sur l'accès à ces mythes en tant que textes anciens (ce qui est appelé "décryptage»), sur la compréhension que les destinataires (Polynésiens d'autrefois, contemporains et Occidentaux) peuvent en nourrir serait éclairante, tant d'un point de vue de méthodologie générale que pour mettre en garde les lecteurs métropolitains. Le passage d'une référence à l'autre, par exemple, sur la base d'idées, de concepts ou de motifs considérés comme proches ou identiques par l'auteur - parfois même les raccourcis permettant les conclusions qu'il en tire - peut être déroutant pour les non-spécialistes de mythologie comparée et ce, quand bien même ils servent un propos convainquant. À titre d'exemple, je retiendrai le problème que pose la grande polysémie du mot «terre » en français et des nombreux termes vernaculaires auquel il renvoie dans les textes et leurs analyses, sans que les distinctions qu'opèrent les langues polynésiennes soient toujours relevées ${ }^{7}$. Force est de constater que nous manquent l'expertise de Bruno Saura et les principes de son herméneutique pour bien saisir, avec la complexité sémantique de certains termes-clés, la nature et le statut initial des textes mobilisés, leurs implicites et les logiques sousjacentes (formelles, sémantiques, rituelles, éventuellement politiques...) propres aux récits anciens, souvent hétérogènes, qui ont été conservés et aux corpus dont ils sont issus.

Ma première remarque porte sur l'usage de catégories préconstruites qui, telles que l'« autochtonie ", peut biaiser l'analyse et gêner la compréhension dans l'étude de faits sociaux relevant d'un tout autre univers social et culturel. Par exemple, il est intéressant que, selon l'auteur :

« [une bonne part des] récits affirment indirectement que l'humanité polynésienne n'est pas autochtone de ces îles, puisque les îles pêchées et peuplées par les groupes humains ne se confondent pas avec la terre Papa » (pp. 130 et 134)

Or, l'auteur a rappelé, en citant Bachimon (1995 : 232), que :

« [d]ans la tradition cosmogonique [polynésienne], c'est invariablement l'humanité qui découvre sa terre. Si l'on excepte le prototype terrien modelé [la création de l'univers] par Taatoa, toutes les autres terres naissent d'un projet, celui d'un demidieu ou d'un héros, qui se propose d'y installer une population. » (p. 125)

Certes, le lien entre les hommes et la terre dont parle Bachimon ne correspond pas à celui retenu pour organiser l'analyse (consubstantialité entre hommes et la terre), mais ne peut-on les considérer comme une alternative ? La création par les dieux d'une terre à destination d'un groupe particulier (ou la découverte de sa terre par un groupe humain) ne revient-elle pas à une affirmation très forte du lien entre la terre et les hommes auxquels elle a été initialement destinée? Une définition différente - et 
localement attestée cette fois - de l'autochtonie se dessinerait ainsi qui, bien que mentionnée, n'est pas retenue comme pertinente dans cet ouvrage.

Aussi l'auteur reconnaît-il de bonne foi, en conclusion, que les mythes, tout comme les faits anciens qui nous sont connus par ailleurs, ne se plient pas - ou mal - aux définitions a priori de l'autochtonie dans ses dimensions contemporaines, notamment telles qu'elles sont définies en introduction. On peut alors questionner la pertinence du concept d'«autochtonie littérale" pour entreprendre l'analyse des anciens mythes polynésiens et la nécessité que cette forme d'autochtonie soit solidement fondée sur des traditions attestées pour qu'elle soit politiquement recevable. Ancienne ou pas, toute idéologie essentialisante est politiquement dangereuse, mais il me semble qu'elle ne saurait être plus ou moins "polynésienne ". Après tout, l'autochtonie littérale des nationalistes relève du mythe et mériterait d'être abordée comme telle.

Une remarque peut aussi être faite sur la théorie sous-jacente aux propositions relatives aux "substances ». Ne conviendrait-il pas plutôt de distinguer des types de relations? L'analyse des récits insiste en effet à de nombreuses reprises, sur la «matérialité » du principe chtonien, de «la Terre» Te Papa et de la matrice qu'elle constitue. L'auteur adopte l'idée selon laquelle les humains, parce qu'ils sont issus de la terre, lui sont consubstantiels, c'est-à-dire qu'ils en partagent la matière. Dans le second chapitre, par exemple (p.63), l'auteur indique que les hommes sont seuls à combiner cette matérialité au caractère spirituel propre aux déités dont ils procèdent, par procréation ou par façonnement (ce qui est infirmé au chapitre précédent puisque des héros et des demi-dieux résultent de mêmes processus). Ce qui me semble important dans la référence à la «terre » et dans l'«appréhension dynamique des essences, des [différentes] matières » (p. 74) qui forme cette catégorie hétéroclite, c'est que les relations passant par un attachement au sol (souvent pensées comme maternelles) n'équivalent pas celles (valorisées et valorisantes) acquises par l'ascendance paternelle et masculine. Passer d'une interprétation en termes de substances à une interprétation en termes de relations implique un changement de paradigme qui affranchit l'analyse des oppositions binaires (matière/esprit, p. 63) si prégnantes dans la pensée occidentale. Ainsi l'humanité, tout au moins en Polynésie occidentale, semble-t-elle se définir, non par la matière (les dieux et les ancêtres ont des formes d'existence matérielle eux aussi), mais par l'agencement particulier de relations. Ces dernières la dotent d'un corps où la vie pulse au sein d'une masse sanglante avant de rejoindre le sol de la sépulture, et de relations pérennes à l'Au-delà, autrefois propres aux aristocrates qui ainsi ne disparaissaient pas dans la mort - depuis l'évangélisation, ces relations pérennes sont générales parmi les chrétiens (je l'ai en tous cas montré pour Wallis, Chave-Dartoen, 2000). Les mythes tongiens et samoans ne me semblent pas dire autre chose lorsqu'ils situent l'origine de l'humanité dans des vers, issus de la pourriture (de la vie surgie des gonflements de la putréfaction), mais aussi d'une relation triple, à la terre, à l'espace social et à l'Au-delà céleste, via les végétaux dont ils sont issus et qui, en s'élevant, font le lien entre la terre et le ciel (p. 72). D'autres mythes me semblent encore dire la même chose lorsqu'ils distinguent les hommes nés sous forme de nourrissons et le placenta, masse sanglante qui, double incomplet du nourrisson, peut être transformée en entité hybride si elle est complétée, en mer, par une relation créée avec les déités qui y résident (p. 66). Ici, la terre, les déités et les autres entités du cosmos dotent les humains des relations (non des substances) nécessaires à leur existence: des mères, des pères, des conjoints, des 
descendances nécessaires à la constitution (p. 71-72) et au déploiement d'un espace qui, s'ouvrant progressivement, les affranchit des relations incestueuses (car indistinctes), encore non sociales, pour se constituer comme un domaine du cosmos ayant ses propres principes et un ordre défini.

La dernière remarque sera relative au lieu d'origine, dont les noms (Pulotu/Hawaiki) pourraient ne pas renvoyer à une île d'où serait partie la migration originelle des Polynésiens, mais, comme l'auteur nous incite à le penser, le lieu à partir duquel chaque société locale pense s'être projetée, lieu à partir duquel s'est déployé son cosmos (p. 34), où résident ses déités ancestrales, et vers lequel voyagent ses défunts. Hawaiki - et ses variations phonologiques - désignerait ainsi, pour chaque société prise séparément, non pas tant un «lieu idéalisé d'où l'on aurait été chassé par des guerres meurtrières ", comme le propose C. Moyse-Faurie (2006: 143), mais le point de référence fondé historiquement et devenu mythique et rituel : Sava'i pour Upolu, et le reste de l'archipel samoan ; Sava'i (et Upolu, de Samoa) pour Ra'iatea et les îles sous le vent; Havai'i (Raiatea) pour Tahiti (cf. notamment pp. 128, 129, 131 et 139) ; l'île de Hawai'i pour le reste de l'archipel hawai'ien'.

Je n'ai pas la place ici pour discuter davantage les innombrables pistes initiées par ce livre. Mes remarques conclusives visent à éclairer ce qui apparait à l'ethnologue soit comme des angles morts, soit comme les ouvertures d'un ouvrage dense et passionnant. Elles ne retirent rien à son intérêt, à sa valeur et à sa dimension utilement critique.

\section{BIBLIOGRAPHIE}

BACHIMON Philippe, 1995. L'insularité océanienne dans la cosmogonie māohi, Espace géographique 24 (3), pp. 227-235.

BAMBRIDGE Tamatoa, Pierre-Yves Le MEUR et Christian JOST, 2016. Représentations polynésiennes, pratiques culturelles et usages sociaux de la ressource et de son environnement, Papeete-Marseille, Au vent des îles/IRD Éditions.

CHAVE-DARTOEN Sophie, 2000. Uvea (Wallis), une société de Polynésie occidentale, étude et comparaison, thèse de doctorat en Ethnologie et Anthropologie sociale, Paris, EHESS.

MOYSE-FAURIE Claire, 2006. Histoires de Pulotu, Journal de la Société des Océanistes 122-123, pp. 141-151 (https://jso.revues.org/603).

SAURA Bruno, 2000. Le placenta en Polynésie française : choix de santé publique et identité, Sciences sociales et santé 18, pp. 5-28.

-, 2004. Dire l'autochtonie à Tahiti. Le terme mā'ohi : représentations, controverse et données linguistiques, Journal de la société des Océanistes 119, pp. 119-137 (https://jso.revues.org/126).

-, 2013. Tahiti mā’ohi : Culture, identité, religion et nationalisme en Polynésie française, Papeete, Au vent des îles.

-, 2014a. Politique et religion à Tahiti, Papeete, Au vent des îles. 
-, 2014b. L'humanité en gestation : figures polynésiennes d'une autochtonie inachevée, Journal de la Société des Océanistes 138-139, pp. 195-208 (https://jso.revues.org/7150).

\section{NOTES}

1. Comme le soulignent T. Bambridge, P.-Y. Le Meur et C. Jost, « [...] une des originalités de la question identitairemā'ohi en Polynésie française, par rapport au reste du Pacifique, réside dans le fait que cette dynamique se fonde essentiellement sur une reconstruction, réelle ou fantasmée, du passé. En effet, après le traumatisme culturel et politique causé par des épidémies de grande ampleur au cours $\mathrm{du} \mathrm{XIX}^{\mathrm{e}}$ et $\mathrm{du} \mathrm{XX}^{\mathrm{e}}$ siècle, une grande partie des savoirs traditionnels a été irrémédiablement perdue. C'est peut-être ce qui rend compte de l'importance en termes d'identité de ce mouvement de réappropriation de la culture et des nouvelles réflexions qui lient culture et religion anciennes et identité contemporaine » (2016: 65).

2. Les deux premiers chapitres, relatifs aux mythes d'autochtonie, s'intitulent « la terre matrice » et la « terre ventre ». Les suivants, sous des titres de même registre (« la terre errante ", « la terre soumise », " la terre matrie »), sont davantage problématisés.

3. L'auteur remarque que le terme a peu d'écho en France (tout comme les Subaltern Studies

), probablement pour cette même raison. La France a développé une politique assimilationniste qui, concernant ce qui reste de ses anciennes colonies, lui réserve une emprise néocoloniale.

4. Symboles, métaphores et métonymiessont parfois relevés parmi les parallèles, les assimilations, les renvois et autres comparaisons... Des procédés tels la « lecture allégorique » (p.150) sont ponctuellement mentionnés.

5. On comprend leur association quand, p. 122, l'auteur indique qu'à Anaa, les créatures inabouties mokorea occupent, selon les mythes, « un monde souterrain » et « un lieu mythique sous la mer ». Certes, la distinction est peu productive du point de vue de l'analyse menée ici, mais elle ne pourrait

être ignorée, en bonne méthode, si l'on cherchait à comprendre ce dont parle chacun des mythes pris séparément.

6. Le propos de ce chapitre a fait l'objet d'une première publication (Saura, 2014b).

7. Alors qu'il traduit le terme par « terre », l'auteur indique, par exemple que papa signifie d'abord « tout élément horizontal plat, socle, niveau, strate, base, fondement, dalle » (p. 27). Il s'agit donc bien d'une surface (p. 37), d'une « plateforme » (p. 78) destinée aux humains, d'un « sol » et non de la « terre » telle que, prévient-il, on l'entend communément (p. 28). Certes, les mythes présentent cette entité papa comme féminine et, parfois comme matricielle. Mais s'agit-il bien de la même « terre » que celle, « nourricière » ('ele'ele) que distingue le mythe commenté pages 70-71 ? Ces questions sémantiques, et notamment la distinction entre papa, 'ele'ele et fenua (" pays » en tahitien, p. 28 et p. 78) et/ou pufenua (p. 85) - entre autres - mériteraient d'être un peu plus documentées (dictionnaires, mythes, gloses) et discutées dans un ouvrage où ces concepts et leurs usages contemporains focalisent l'analyse.

8. Il n'en va pas exactement de même pour Pulotu, qui ne désigne pas une terre ancestrale, mais un domaine du cosmos (une île immergée où résident les déités). Une proposition de P. Geraghty (1993), très séduisante, va toutefois dans ce sens : il situe 
l'origine du nom Borotu àMatuku, dans l'est fidjien, d'où pourraient être originaires les premiers habitants de Samoa, Tonga, Wallis-et-Futuna. Voir Chave-Dartoen (2000) pour une version alternative du mythe wallisien de Pulotu - recueillie auprès du même informateur, mais quelques années avant Moyse-Faurie (2006).

\section{AUTEUR}

\section{SOPHIE CHAVE-DARTOEN}

PASSAGES (UMR 5319 cNRS-Université de Bordeaux 